\title{
THE AUTOMORPHISMS OF GENERALIZED CYCLIC AZUMAYA ALGEBRAS
}

\author{
S. PUMPLÜN
}

\begin{abstract}
We define a nonassociative generalization of cyclic Azumaya algebras employing skew polynomial rings $D[t ; \sigma]$, where $D$ is an Azumaya algebra of constant rank with center $C$ and $\sigma$ an automorphism of $D$, such that $\left.\sigma\right|_{C}$ has finite order. The automorphisms of these algebras are canonically induced by ring automorphisms of the skew polynomial ring $D[t ; \sigma]$ used in their construction. We achieve a description of their (left) inner automorphisms. Results on the automorphisms of classical Azumaya algebras and central simple algebras of this type are obtained as special cases.
\end{abstract}

\section{INTRODUCTION}

Let $D$ be an Azumaya algebra of constant rank with center $C$. Let $\sigma \in \operatorname{Aut}(D)$ be a ring automorphism, such that $\left.\sigma\right|_{C}$ has finite order $m$ and fixed $\operatorname{ring} S_{0}=\operatorname{Fix}(\sigma) \cap C$. We assume that $C / S_{0}$ is a cyclic Galois ring extension of degree $m$ with Galois group $\operatorname{Gal}\left(C / S_{0}\right)=\left\langle\left.\sigma\right|_{C}\right\rangle$. We define generalized cyclic Azumaya algebras with the help of the skew polynomial rings $D[t ; \sigma]$ as quotient algebras $D[t ; \sigma] / D[t ; \sigma] f$ for some $f(t)=t^{m}-d$, where $m$ is the order of $\left.\sigma\right|_{C}$ and $d \in S_{0}=C \cap \operatorname{Fix}(\sigma)$. Generalized cyclic Azumaya algebras are special examples of crossed product algebras that are Azumaya algebras: the crossed product is taken using $D$ and the cyclic group $\langle\sigma\rangle$. In particular, let $\sigma$ be an automorphism of $S$ with fixed ring $S_{0}$, and let $S / S_{0}$ be a Galois ring extension with cyclic Galois group $G=\langle\sigma\rangle$. Then this construction yields cyclic Azumaya algebras.

This approach allows us to fit generalized Azumaya algebras into a more general family of nonassociative algebras. In order to do so we use a construction that goes back to Petit [17]. We note that the skew polynomials $R_{m}=\{h \in D[t ; \sigma] \mid \operatorname{deg}(h)<m\}$ of degree less than $m$ canonically represent the elements of the left $D[t ; \sigma]$-module $D[t ; \sigma] / D[t ; \sigma] f$, for any $f \in D[t ; \sigma]$ of degree $m$. Let $f(t)=t^{m}-d$, where $m$ is the order of $\left.\sigma\right|_{C}$, but allow $d \in D^{\times}$. Define the new $S_{0}$-algebra $S_{f}$ on the additive subgroup $R_{m}$ by using right division by $f$ to define the algebra multiplication $g \circ h=g h \bmod _{r} f$. This algebra is not associative for $d \notin S_{0}$, but coincides with the associative quotient algebra $D[t ; \sigma] / D[t ; \sigma] f$ if $d \in S_{0}$. The algebras $S_{f}$ were introduced and studied in detail by Petit in $[17,18]$ when $D$ is a division ring, and more generally for arbitrary rings in [19].

Note that associative generalized cyclic algebras over fields were investigated by Amitsur in [3], and nonassociative generalized cyclic algebras over fields in [7]. Another generalization

2010 Mathematics Subject Classification. Primary: 17A35; Secondary: 17A60, 16S36.

Key words and phrases. Skew polynomial, Ore polynomial, Azumaya algebra, cyclic algebra. 
of associative generalized cyclic algebras over fields called associative cyclic extensions of simple rings was considered by Kishimoto [14].

After introducing the basic terminology in Section 1, we introduce nonassociative generalized cyclic Azumaya algebras and some of their properties in Section 2. In Section 3, we then prove that every automorphism of a nonassociative generalized Azumaya algebra $A$ of constant rank over $S_{0}$ is canonically induced by some ring automorphism of $D[t ; \sigma]$ (Theorem 10). Furthermore, the automorphisms $H_{i d_{D}, k}$ that extend $i d_{D}$ are in one-one correspondence with the elements of the group $\left\{c \in C \mid N_{C / S_{0}}(c)=1\right\}$. These are the only automorphisms of $A$, unless some $\tau \neq i d_{D}$ that commutes with $\sigma$ can be extended to an $S_{0^{-}}$ automorphism of $A$ as well. In particular, every automorphism of an associative generalized cyclic Azumaya algebra $A$ of constant rank extends an automorphism $\tau \in \operatorname{Aut}_{S_{0}}(D)$ that commutes with $\sigma$, and the possible extensions $H_{\tau, k}$ of an $S_{0}$-automorphism $\tau$ that commutes with $\sigma$ are in one-one correspondence with the group $\left\{c \in C \mid N_{C / S_{0}}(c)=1\right\}$. Moreover, the $S_{0}$-automorphisms $\tau$ of $D$ that commute with $\sigma$ form a subgroup of the automorphism group of the Azumaya algebra.

In Section 4, we show that all automorphisms of a nonassociative generalized cyclic Azumaya algebra can be written as a composition of an inner automorphism and a map that is a canonical extension of $\tau$ to $A$ of the type $\sum_{i=0}^{m-1} a_{i} t^{i} \mapsto \sum_{i=0}^{m-1} \tau\left(a_{i}\right) t^{i}$ (Theorem 16). We also prove that the automorphisms $H_{i d_{D}, k}$ of a nonassociative generalized cyclic Azumaya algebra which extend the identity $i d_{D}$ are inner for all $k \in C$, such that there is $c \in C^{\times}$with $k=c^{-1} \sigma(c)$. In the special case that we have a nonassociative generalized cyclic algebra $(D, \sigma, d)$ over a base field, all its automorphisms are of this last type.

As an immediate consequence of our results, the automorphisms of an Azumaya algebra $(D, \sigma, d)$, where the ring extension satisfies an analogue of Hilbert's Theorem 90, are the composition of an inner automorphism $G_{c}, c \in C^{\times}$with the canonical extension $H_{\tau, 1}$ of some $\tau \in \operatorname{Aut}_{S_{0}}(D)$ which commutes with $\sigma$ (Corollary 18). In particular, the automorphisms of a generalized cyclic central simple algebra over a field are induced by ring automorphisms of the ring $D[t ; \sigma]$ used in their construction; each is the composition of an inner automorphism $G_{c}, c \in C^{\times}$with the canonical extension $H_{\tau, 1}$ of some $\tau \in \operatorname{Aut}_{S_{0}}(D)$ which commutes with $\sigma$ (Corollary 19). If $A=(K / F, \sigma, d)$ is an associative cyclic central simple algebra, we show that all its automorphism can be described as inner automorphisms of the type $G_{c t^{j}}$ for some $c \in K$ with $N_{K / F}(c)=1$ and a suitable integer $j, 0 \leq j \leq m-1$.

\section{Preliminaries}

1.1. Nonassociative algebras. Let $R$ be a unital commutative ring and let $A$ be an $R$ module. $A$ is an algebra over $R$ if there exists an $R$-bilinear map $A \times A \rightarrow A,(x, y) \mapsto x \cdot y$, denoted simply by juxtaposition $x y$, the multiplication of $A$. An algebra $A$ is called unital if there is an element in $A$, denoted by 1 , such that $1 x=x 1=x$ for all $x \in A$. We will only consider unital algebras.

The associator of $A$ is given by $[x, y, z]=(x y) z-x(y z)$. The left nucleus of $A$ is defined as $\operatorname{Nuc}_{l}(A)=\{x \in A \mid[x, A, A]=0\}$, the middle nucleus of $A$ is $\operatorname{Nuc}_{m}(A)=\{x \in A \mid[A, x, A]=$ $0\}$ and the right nucleus of $A$ is $\operatorname{Nuc}_{r}(A)=\{x \in A \mid[A, A, x]=0\}$. $\operatorname{Nuc}_{l}(A), \operatorname{Nuc}_{m}(A)$, and 
$\operatorname{Nuc}_{r}(A)$ are associative subalgebras of $A$. Their intersection $\operatorname{Nuc}(A)=\{x \in A \mid[x, A, A]=$ $[A, x, A]=[A, A, x]=0\}$ is the nucleus of $A . \operatorname{Nuc}(A)$ is an associative subalgebra of $A$ containing $F 1$ and $x(y z)=(x y) z$ whenever one of the elements $x, y, z$ lies in $\operatorname{Nuc}(A)$. The center of $A$ is defined as $\mathrm{C}(A)=\{x \in A \mid x \in \operatorname{Nuc}(A)$ and $x y=y x$ for all $y \in A\}$. Define $\operatorname{Comm}(A)=\{x \in A \mid x y=y x$ for all $y \in A\}$. For a subring $B$ of a unital ring $A$, the centralizer (also called the commutator subring if $A$ is associative) of $B$ in $A$ is defined as $\operatorname{Cent}_{A}(B)=\{a \in A \mid a b=b a$ for all $b \in B\}$. If $A$ is not associative, $\operatorname{Cent}_{A}(B)$ need not be a ring. If $\operatorname{Cent}_{A}(B)=B$ then $\operatorname{Cent}_{A}(B)$ is a maximal commutative nonassociative subring of $A$.

An automorphism $G \in \operatorname{Aut}(A)$ of an algebra $A$ is called a (left) inner automorphism if there is an element $m \in A$ with left inverse $m_{l}$ such that $G(x)=\left(m_{l} x\right) m$ for all $x \in A$. We denote such an automorphism by $G_{m}$. It is possible to define (right) inner automorphisms analogously as $F_{m}(x)=m\left(x m_{r}\right)$ for all $x \in A$, if there is an element $m \in A$ with right inverse $m_{r}$, but these are not needed in this paper. Given an inner automorphism $G_{m} \in \operatorname{Aut}(A)$ and some $H \in \operatorname{Aut}(A), H^{-1} \circ G_{m} \circ H \in \operatorname{Aut}(A)$ is also an inner automorphism. Indeed, $[27$, Lemma 2 , Theorem 3,4$]$ generalize to any nonassociative algebra over a ring:

Proposition 1. Let $A$ be an algebra over $R$.

(i) For all invertible $n \in \operatorname{Nuc}(A), G_{n}(x)=\left(n^{-1} x\right) n$ is an inner automorphism of $A$.

(ii) If $G_{m}$ is an inner automorphism of $A$, then so is $G_{n m}(x)=\left(\left(m_{l} n^{-1}\right) x\right)(n m)$ for all invertible $n \in \operatorname{Nuc}(A)$.

(iii) If $G_{m}$ is an inner automorphism of $A$, and $a, b \in \operatorname{Nuc}(A)$ are invertible, then $G_{a m}=$ $G_{b m}$ if and only if $a b^{-1} \in C(A)$.

(iv) For invertible $n, m \in \operatorname{Nuc}(A), G_{m}=G_{n}$ if and only if $n^{-1} m \in C(A)$.

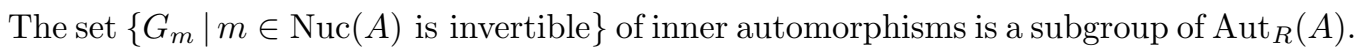
For each invertible $m \in \operatorname{Nuc}(A) \backslash C(A), G_{m}$ generates a cyclic subgroup of inner automorphisms which has finite order $s$ if $m^{s} \in C(A)$, so in particular if $m$ has order $s$.

If the nucleus of $A$ is commutative, then for all invertible $n \in \operatorname{Nuc}(A), G_{n}(x)=\left(n^{-1} x\right) n$ is an inner automorphism of $A$ such that $\left.G_{n}\right|_{\mathrm{Nuc}(A)}=i d_{\mathrm{Nuc}(A)}$.

1.2. Azumaya algebras. An algebra $A$ is called an Azumaya algebra over a unital ring $R$, if $A$ is finitely generated as an $R$-module, $A$ is a separable extension of $R$, and the center of $A$ is $R$. Equivalently, $A$ is an Azumaya algebra if $A$ is a finitely generated $R$-module and $A / A m$ is a central simple $R / m$-algebra, for all maximal ideals $m$ in $R$.

A commutative ring extension $R^{\prime}$ of $R$ is called a splitting ring of $A$ if $A \otimes_{R} R^{\prime} \cong \operatorname{End}_{R^{\prime}}(P)$ for a suitable faithfully projective $R^{\prime}$-module $P$.

If $S$ is a maximal commutative subalgebra in $A$ and $S$ is separable over $R$ then $S$ is a splitting ring for $A[15]$.

1.3. Galois extensions. Let $S$ be a commutative ring. Recall that two $S_{0}$-algebra homomorphisms $\sigma$ and $\tau$ are called strongly distinct, if for every non-zero idempotent $e \in S$ there is $x \in S$ such that $\sigma(x) e \neq \tau(x) e$.

If we assume that $S$ is an $S_{0}$-algebra and faithfully projective as an $S_{0}$-module, and that $G$ is a group of $S_{0}$-algebra homomorphisms of $S$, then the following conditions are equivalent: 
(i) $S$ is a separable $S_{0}$-algebra, finitely generated projective as an $S_{0}$-module, and $\operatorname{rank}_{S_{0}} S=$ $|G|$. All elements of $G$ are pairwise strongly distinct.

(ii) $S$ is faithfully projective as an $S_{0}$-module, $\operatorname{rank}_{S_{0}} S=|G|$ and for every $\sigma \in G$ there exist $x_{i, \sigma}, y_{i, \sigma} \in S$ such that $\sum_{i=1}^{m_{\sigma}} x_{i, \sigma} \tau\left(y_{i, \sigma}\right)=\delta_{\tau, \sigma}$ for all $\tau \in G$.

(iii) $\varphi: S \otimes S \longrightarrow S^{n}, \varphi(s \otimes t)=(s \tau(t))_{\tau \in G}$ is an isomorphism of $S$-algebras where $n$ is the order of $G$.

If one of these conditions is satisfied we call $S / S_{0}$ a Galois extension with Galois group $G$ in the sense of Chase-Harrison-Rosenberg [9].

Moreover, $S / S_{0}$ is called a weakly Galois extension (resp., (G-)Galois in Szeto's papers $[22,23,24,25])$ if $S$ is a separable algebra over $S_{0}$, finitely generated projective as $S_{0}$-module, and there is a finite group of automorphisms $G$ of $S$ such that $S_{0}=S^{G}$ is the fixed ring of $G$ in $S$. Note that if $S / S_{0}$ is a Galois extension with Galois group $G$ then this implies that $S^{G}=S_{0}$. If $S / S_{0}$ is a Galois extension and the elements in $G$ are $S_{0}$-automorphisms of $S$ then $S$ is a weakly Galois extension of $S_{0}$ of constant rank (cf. [11, Lemma 2.3, Corollary 2.4] for this summary).

If $S / S_{0}$ is a Galois extension with Galois group $G$, then the map $H \mapsto S^{H}$ gives a one-one correspondence between the set of subgroups of $G$ and the set of $S_{0}$-subalgebras $S_{H}$ of $S$ which are separable and $G$-strong, i.e. the restriction of any two elements of $G$ to $S_{H}$ are either equal or strongly distinct as maps from $S_{H}$ to $S_{H}$.

In this paper, we will use commutative Galois extensions that are weakly commutative Galois extensions.

1.4. Skew polynomial rings. Let $S$ be a unital associative ring, $\sigma$ a ring endomorphism of $S$ and $\delta: S \rightarrow S$ a left $\sigma$-derivation, i.e. an additive map such that $\delta(a b)=\sigma(a) \delta(b)+\delta(a) b$ for all $a, b \in S$. Then the skew polynomial ring $S[t ; \sigma, \delta]$ is the set of skew polynomials $g(t)=a_{0}+a_{1} t+\cdots+a_{n} t^{n}$ with $a_{i} \in S$, with term-wise addition and where the multiplication is defined via $t a=\sigma(a) t+\delta(a)$ for all $a \in S$. That means,

$$
a t^{n} b t^{m}=\sum_{j=0}^{n} a\left(\Delta_{n, j} b\right) t^{m+j}
$$

for all $a, b \in S$, where the map $\Delta_{n, j}$ is defined recursively via

$$
\Delta_{n, j}=\delta\left(\Delta_{n-1, j}\right)+\sigma\left(\Delta_{n-1, j-1}\right)
$$

with $\Delta_{0,0}=i d_{S}, \Delta_{1,0}=\delta, \Delta_{1,1}=\sigma$. Therefore $\Delta_{n, j}$ is the sum of all polynomials in $\sigma$ and $\delta$ of degree $j$ in $\sigma$ and degree $n-j$ in $\delta$ [13, p. 2]. If $\delta=0$, then $\Delta_{n, n}=\sigma^{n}$.

For $\sigma=i d$ and $\delta=0$, we obtain the usual ring of left polynomials $S[t]=S[t ; i d, 0]$. Define $\operatorname{Fix}(\sigma)=\{a \in S \mid \sigma(a)=a\}$ and $\operatorname{Const}(\delta)=\{a \in S \mid \delta(a)=0\}$.

For $f(t)=a_{0}+a_{1} t+\cdots+a_{n} t^{n} \in S[t ; \sigma, \delta]$ with $a_{n} \neq 0$ define $\operatorname{deg}(f)=n$ and $\operatorname{deg}(0)=-\infty$. Then $\operatorname{deg}(g h) \leq \operatorname{deg}(g)+\operatorname{deg}(h)$ (with equality if $h$ has an invertible leading coefficient, or $g$ has an invertible leading coefficient and $\sigma$ is injective, or if $S$ is a division ring). An element $f \in S[t ; \sigma, \delta]$ is irreducible if it is not a unit and it has no proper factors, i.e if there do not exist $g, h \in R$ such that $f=g h$. 
1.5. Algebras obtained from skew polynomial rings. Let $S$ be a unital associative ring and $R=S[t ; \sigma, \delta]$ a skew polynomial ring where $\sigma$ is injective.

Assume $f(t)=\sum_{i=0}^{m} a_{i} t^{i} \in S[t ; \sigma, \delta]$ has an invertible leading coefficient $a_{m} \in S^{\times}$. Then for all $g(t) \in S[t ; \sigma, \delta]$ of degree $l \geq m$, there exist uniquely determined $r(t), q(t) \in S[t ; \sigma, \delta]$ with $\operatorname{deg}(r)<\operatorname{deg}(f)$, such that $g(t)=q(t) f(t)+r(t)([6],[19$, Proposition 1]).

Let $\bmod _{r} f$ denote the remainder of right division by $f$. The skew polynomials of degree less that $m$ canonically represent the elements of the right $S[t ; \sigma, \delta]$-module $S[t ; \sigma, \delta] / S[t ; \sigma, \delta] f$. Moreover,

$$
\{g \in S[t ; \sigma, \delta] \mid \operatorname{deg}(g)<m\}
$$

together with the multiplication

$$
g \circ h=\left\{\begin{array}{l}
g h \text { if } \operatorname{deg}(g)+\operatorname{deg}(h)<m, \\
g h \bmod _{r} f \text { if } \operatorname{deg}(g)+\operatorname{deg}(h) \geq m,
\end{array}\right.
$$

is a unital nonassociative ring $S_{f}$ also denoted by $S[t ; \sigma, \delta] / S[t ; \sigma, \delta] f . S_{f}$ is a unital nonassociative algebra over the commutative subring

$$
\left\{a \in S \mid a h=h a \text { for all } h \in S_{f}\right\}=\operatorname{Comm}\left(S_{f}\right) \cap S
$$

of $S$. When the context is clear, we will drop the o notation and simply use juxtaposition for multiplication in $S_{f}$. Note that if $f$ has degree 1 then $S_{f} \cong S$, and if $f$ is reducible then $S_{f}$ contains zero divisors. For all invertible $a \in S$ we have $S_{f}=S_{a f}$.

In the following, we assume $m \geq 2$ and call the algebras $S_{f}$ Petit algebras as the construction goes back to Petit [17].

$S_{f}$ is a free left $S$-module of rank $m$ with basis $t^{0}=1, t, \ldots, t^{m-1} . S_{f}$ is associative if and only if $R f$ is a two-sided ideal in $R$. If $S_{f}$ is not associative then $S \subseteq \operatorname{Nuc}_{l}\left(S_{f}\right), S \subseteq$ $\operatorname{Nuc}_{m}\left(S_{f}\right)$ and

$$
\{g \in R \mid \operatorname{deg}(g)<m \text { and } f g \in R f\}=\operatorname{Nuc}_{r}\left(S_{f}\right) .
$$

When $S$ is a division ring, these inclusions become equalities [19, Theorem 4].

Note that $C\left(S_{f}\right)=\operatorname{Comm}\left(S_{f}\right) \cap \operatorname{Nuc}_{l}\left(S_{f}\right) \cap \operatorname{Nuc}_{m}\left(S_{f}\right) \cap \operatorname{Nuc}_{r}\left(S_{f}\right)$ and so

$$
\left\{a \in S \mid a h=h a \text { for all } h \in S_{f}\right\}=\operatorname{Comm}\left(S_{f}\right) \cap S \subseteq C\left(S_{f}\right) .
$$

If $\operatorname{Nuc}_{l}\left(S_{f}\right)=\operatorname{Nuc}_{m}\left(S_{f}\right)=S$ this yields that the center $C\left(S_{f}\right)=\operatorname{Comm}\left(S_{f}\right) \cap S \cap$ $\operatorname{Nuc}_{r}\left(S_{f}\right)=\operatorname{Comm}\left(S_{f}\right) \cap S$ of $S_{f}$ is identical to the ring $\left\{a \in S \mid a h=h a\right.$ for all $\left.h \in S_{f}\right\}$. Also note that

$$
C(S) \cap \operatorname{Fix}(\sigma) \cap \operatorname{Const}(\delta) \subseteq\left\{a \in S \mid a h=h a \text { for all } h \in S_{f}\right\}
$$

which is proved analogously as [19, Theorem 8 (ii)], where $\delta=0$.

If $\delta=0$ and $f(t)=\sum_{i=0}^{m} a_{i} t^{i} \in S[t ; \sigma]$ then $\operatorname{Comm}\left(S_{f}\right)=\left\{g \in S_{f} \mid g h=h g\right.$ for all $h \in$ $\left.S_{f}\right\}$ contains the set

$$
\left\{\sum_{i=0}^{m-1} d_{i} t^{i} \mid d_{i} \in \operatorname{Fix}(\sigma) \text { and } c d_{i}=d_{i} \sigma^{i}(c) \text { for all } c \in S\right\}
$$


[19, Theorem 8 (i)]. If $a_{0}$ is invertible, the two sets are equal [6, Proposition 7.4 (iii)], implying

$\left\{a \in S \mid a h=h a\right.$ for all $\left.h \in S_{f}\right\}=\{a \in S \mid a \in \operatorname{Fix}(\sigma)$ and $c a=a c$ for all $c \in S\}=C(S) \cap \operatorname{Fix}(\sigma)$.

\section{Nonassociative generalized Cyclic Azumaya Algebras}

In the following, let $D$ be a unital ring with center $C=C(D)$. Let $\sigma \in \operatorname{Aut}(D)$ be a ring automorphism, such that $\left.\sigma\right|_{C}$ has finite order $m$ and fixed ring $S_{0}=\operatorname{Fix}(\sigma) \cap C$. Moreover, we assume that $C / S_{0}$ is a cyclic Galois ring extension of degree $m$ with Galois group $\operatorname{Gal}\left(C / S_{0}\right)=\left\langle\left.\sigma\right|_{C}\right\rangle$. This implies that $C$ has constant rank $m$ as an $S_{0}$-module.

Let $f(t)=t^{m}-d \in D[t ; \sigma]$ with $d \in D^{\times}$invertible. Then $S_{f}=D[t ; \sigma] / D[t ; \sigma]\left(t^{m}-d\right)$ is a nonassociative algebra over its center

$$
\left\{a \in D \mid a h=h a \text { for all } h \in S_{f}\right\}=C \cap \operatorname{Fix}(\sigma)=S_{0} .
$$

Moreover, since $d$ is invertible we know that

$$
\operatorname{Comm}\left(S_{f}\right)=\left\{\sum_{i=0}^{m-1} d_{i} t^{i} \mid d_{i} \in \operatorname{Fix}(\sigma) \text { and } c d_{i}=d_{i} \sigma^{i}(c) \text { for all } c \in S\right\} .
$$

In the associative setting, i.e. when $d \in S_{0}^{\times}$, we have the following result:

Theorem 2. Let $S_{f}=D[t ; \sigma] / D[t ; \sigma]\left(t^{m}-d\right)$ with $d \in S_{0}^{\times}$. Then the following statements are equivalent:

(i) $D[t ; \sigma] / D[t ; \sigma]\left(t^{m}-d\right)$ is an Azumaya algebra over $S_{0}$.

(ii) $D$ is an Azumaya algebra over $C$.

Proof. (ii) implies (i): Since $D$ is an Azumaya algebra over $C, D$ is separable over $C$. Moreover, $S_{f}$ is a separable extension of $D$, since

$$
x=m^{-1} d^{-1} \sum_{i=1}^{m-1} t^{i} \otimes t^{m-1-i}
$$

is a separable idempotent of $S_{f}$ over $D$ : we have $m^{-1} d^{-1} \sum_{i=1}^{m-1} t^{i} \otimes_{D} t^{m-1-i}=1$ and $g x=x g$ for all $g \in S_{f}$. Thus $S_{f}$ is a separable extension of $S_{0}$ by the transitivity of separable extensions. By our general theory on Petit algebras, we know that $S_{f}$ is an algebra with center $S_{0}$. By construction, $S_{f}$ is finitely generated as an $S_{0}$-module, since $D$ is finitely generated over $C$. Therefore $S_{f}$ is an Azumaya algebra over $S_{0}$.

(i) implies (ii): Assume that $D[t ; \sigma] / D[t ; \sigma]\left(t^{m}-d\right)$ is an Azumaya algebra. Then its center is $S_{0}=C \cap \operatorname{Fix}(\sigma)$. By Proposition 6 below, the centralizer $\operatorname{Cent}_{(D, \sigma, d)}(C)$ of $C$ in $(D, \sigma, d)$ is $D$. Thus $D$ is a separable $S_{0}$-algebra by the Commutator Theorem for Azumaya algebras [12, Theorem 4.3], since $C$ is a separable $S_{0}$-algebra. Therefore $D$ is an Azumaya algebra.

Assuming additionally that $\sigma$ has finite order $m$, we obtain:

Theorem 3. Let $S_{f}=D[t ; \sigma] / D[t ; \sigma]\left(t^{m}-d\right)$ with $d \in S_{0}^{\times}$and let $\sigma$ have finite order $m$. Then the following statements are equivalent:

(i) $D[t ; \sigma] / D[t ; \sigma]\left(t^{m}-d\right)$ is an Azumaya algebra over $S_{0}$. 
(ii) Let $G_{t}(g)=t g t^{-1}$ be the inner automorphism of $S_{f}$ defined by $t$. Then $\left\{u \in S_{f} \mid G_{t}(u)=\right.$ $u\}=\operatorname{Fix}(\sigma)[t] / \operatorname{Fix}(\sigma)[t]\left(t^{m}-d\right)$ is an Azumaya algebra with center $S_{0}[t] / S_{0}[t]\left(t^{m}-d\right)$.

Proof. We only need to show that $\left\{u \in S_{f} \mid G_{t}(u)=u\right\}=\operatorname{Fix}(\sigma)[t] / \operatorname{Fix}(\sigma)[t]\left(t^{m}-d\right)$. The rest is proved in [22, Theorem 3.3]. For this we note that both $\operatorname{Fix}(\sigma) \subseteq\left\{u \in S_{f} \mid G_{t}(u)=u\right\}$ and $t, t^{2}, \ldots, t^{m-1} \in\left\{u \in S_{f} \mid G_{t}(u)=u\right\}$. Moreover, we have $t^{m}=d \in\left\{u \in S_{f} \mid G_{t}(u)=\right.$ $u\}$, so that $\left\{u \in S_{f} \mid u(t) \in \operatorname{Fix}(\sigma)[t]\right\} \subseteq \operatorname{Fix}(\sigma)[t]$. This proves the assertion.

More generally, we still have:

Theorem 4. Let $D$ be an Azumaya algebra over $C$ and let $\left.\sigma\right|_{C}$ have finite order $m$. Consider the Azumaya algebra $S_{f}=D[t ; \sigma] / D[t ; \sigma]\left(t^{m}-d\right), d \in S_{0}^{\times}$. Let $G_{t}(g)=t g t^{-1}$ be the inner automorphism of $S_{f}$ defined by $t$. Then:

(i) $D[t ; \sigma] / D[t ; \sigma]\left(t^{m}-d\right)$ is a $G_{t}$-Galois extension of $S_{f}^{G_{t}}=\left\{u \in S_{f} \mid G_{t}(u)=u\right\}$, such that $S_{f}^{G_{t}}$ is a direct summand of $D[t ; \sigma] / D[t ; \sigma]\left(t^{m}-d\right)$ as a bimodule over $S_{f}^{G_{t}}$.

(ii) $\left\{u \in S_{f} \mid G_{t}(u)=u\right\}=\operatorname{Fix}(\sigma)[t] / \operatorname{Fix}(\sigma)[t]\left(t^{m}-d\right)$ is an Azumaya algebra with center $S_{0}[t] / S_{0}[t]\left(t^{m}-d\right)$.

Proof. (i) The proof of [22, Theorem 3.3] for this statement carries over verbatim. Note that $t$ is invertible in $S_{f}$ with inverse $t^{m-1} d^{-1}$.

(ii) The corresponding part of the proof of [22, Theorem 3.1] does not need the assumption that $\sigma \in \operatorname{Aut}(D)$ has finite order $m$, since we assume that $d \in S_{0}^{\times}$, so in particular that $d \in C$, and we assume that $\left.\sigma\right|_{C}$ has finite order order $m$.

Definition 1. Let $D$ be an Azumaya algebra of constant rank with center $C$ and $f(t)=$ $t^{m}-d \in D[t ; \sigma]$ with $d \in S_{0}^{\times}$. Then the associative algebra $S_{f}=D[t ; \sigma] / D[t ; \sigma] f$ in Theorem 2 is called a generalized cyclic Azumaya algebra. We write $(D, \sigma, d)$ for this algebra.

We follow Jacobson's terminology [13, p. 19]. Note that for $d \in S_{0}^{\times},(D, \sigma, d)$ can also be viewed as a crossed product algebra.

In particular, if $D=C$ is a commutative ring, $C / S_{0}$ is a cyclic Galois extension of degree $m$ with Galois group generated by $\sigma$, and $f(t)=t^{m}-d \in S_{0}[t]$, then we obtain an associative Azumaya algebra we will denote by $\left(C / S_{0}, \sigma, d\right)=C[t, \sigma] / C[t ; \sigma] f$, which we call a cyclic Azumaya algebra. Note that if $C / S_{0}$ is a cyclic field extension, then $\left(C / S_{0}, \sigma, d\right)$ is an associative cyclic algebra over $S_{0}$ of degree $m^{2}$.

Remark 5. (i) This construction of Azumaya algebras was first mentioned in [26] for $f(t)=$ $t^{2}-1 \in D[t ; \sigma]$ with $D$ a commutative ring, as a generalization of the classical quaternion algebra. It was shown that $S_{f}=(D, \sigma, d)$ is an Azumaya algebra over $S_{0}$, that $D$ is a maximal commutative subalgebra of $(D, \sigma, d)$ [25, Lemma 3.1], and that $(D, \sigma, d)$ is a separable extension over $\operatorname{Fix}(\sigma)$. The centralizer of $D$ in $(D, \sigma, d)$ given by $\left\{g \in S_{f} \mid g s=\right.$ $s g$ for all $s \in D\}$ was shown to be $D$. Moreover, $S \otimes_{S_{0}}(D, \sigma, d) \cong \operatorname{Mat}_{n}(S)$. (In [23], $(D, \sigma, d)$ is called a generalized quaternion ring extension.)

(ii) The associative setting in [22] is more restrictive than the one we consider: in [22], the ring automorphism $\sigma \in \operatorname{Aut}(D)$ is always required to have finite order $m$, and $d \in \operatorname{Fix}(\sigma)$. Since $m$ is the order of $\sigma$, in that case $t^{m} b=\sigma^{m}(b) t^{m}=b t^{m}$ for all $b \in D$, which implies 
$d b=b d$ in $S_{f}$ for all $b \in D$, hence $d \in C$, i.e. $d \in C \cap \operatorname{Fix}(\sigma)$.

(iii) If $D$ is a central simple algebra over a field $F$ of degree $n$, then $(D, \sigma, d)$ is a central simple algebra over the field $S_{0}$ of degree $m n$ and the centralizer of $D$ in $(D, \sigma, d)$ is $F[13$, p. 20, Proposition 1.4.4].

(iv) If $m$ is an invertible integer in $D$, then $S_{0}[t] / S_{0}[t]\left(t^{m}-d\right)$ is a separable extension of $S_{0}$ contained in $A=(D, \sigma, d)[22]$.

Our definition of associative generalized cyclic Azumaya algebras generalizes to nonassociative algebras as follows:

Definition 2. Let $D$ be an Azumaya algebra over $C$ and $f(t)=t^{m}-d \in D[t ; \sigma], d \in D^{\times}$. The algebra $(D, \sigma, d)=D[t ; \sigma] / D[t ; \sigma] f$ over $S_{0}$ is called a nonassociative generalized cyclic Azumaya algebra.

In particular, if $D$ has constant rank $n$, then the algebra $A=(D, \sigma, d), d \in D^{\times}$, is finitely generated as an $S_{0}$-module of constant rank $m^{2} n^{2}$.

If $S$ is a commutative ring, and $S / S_{0}$ is a cyclic Galois extension of degree $m$ with Galois group generated by $\sigma$ and $f(t)=t^{m}-d \in S[t ; \sigma]$, we call the algebra $S[t, \sigma] / S[t ; \sigma] f$ a nonassociative cyclic Azumaya algebra and denote it by $\left(S / S_{0}, \sigma, d\right)$ (this is the case where $D=C)$.

The following generalizes [22, Lemma 3.2] to the nonassociative setting:

Proposition 6. For all $d \in D^{\times}$, the centralizer $\operatorname{Cent}_{(D, \sigma, d)}(C)$ of $C$ in $(D, \sigma, d)$ is $D$.

Proof. The proof is analogous to the one in the associative case when $d \in S_{0}^{\times}$: It is easy to see that $D \subseteq \operatorname{Cent}_{(D, \sigma, d)}(C)=\{a(t) \in(D, \sigma, d) \mid a(t) c=c a(t)$ for all $c \in C\}$. Conversely, for each $\sum_{i=0}^{m-1} a_{i} t^{i} \in \operatorname{Cent}_{(D, \sigma, d)}(C)$, we know that $c\left(\sum_{i=0}^{m-1} a_{i} t^{i}\right)=\left(\sum_{i=0}^{m-1} a_{i} t^{i}\right) c$ for all $c \in C$, implying that $a_{i}\left(c-\sigma^{i}(c)\right)=0$ for all $c \in C$ and for all $i$. Since by assumption $C$ is a cyclic Galois extension of $S_{0}$, the ideal of $C$ generated by $\left\{c-\sigma^{i}(c) \mid c \in C\right\}$ is $C$. This means $a_{i}=0$ for all $i>0$ and so $\sum_{i=0}^{m-1} a_{i} t^{i}=a_{0} \in D$, yielding $\operatorname{Cent}_{(D, \sigma, d)}(C) \subseteq D$.

The general structure theory of Petit algebras gives us the following two results:

Theorem 7. Let $\left(S / S_{0}, \sigma, d\right)$ be a nonassociative cyclic Azumaya algebra over $S_{0}$.

(i) $\left(S / S_{0}, \sigma, d\right)$ is finitely generated as an $S_{0}$-module of constant rank $m^{2}$.

(ii) $\left(S / S_{0}, \sigma, d\right)$ is associative if and only if $d \in S_{0}$. If $\left(S / S_{0}, \sigma, d\right)$ is not associative then $\left(S / S_{0}, \sigma, d\right)$ contains $S$ in its left and middle nucleus, and if $S_{0}$ is a domain, then $\operatorname{Nuc}_{l}((D, \sigma, d))=\operatorname{Nuc}_{m}((D, \sigma, d))=S$.

(iii) Let $s$ be the smallest integer such that $d \in \operatorname{Fix}\left(\sigma^{s}\right)$. Then $r s=m$ for some integer $r$ and the finitely generated $S_{0}$-module $S \oplus S t^{s} \oplus \cdots \oplus S t^{(r-1) s}$ of rank $m r$ lies in $\mathrm{Nuc}_{r}\left(\left(S / S_{0}, \sigma, d\right)\right)$. If $s \neq 1$ is a prime or $S_{0}$ is a domain, then $\operatorname{Nuc}_{r}\left(\left(S / S_{0}, \sigma, d\right)\right)=S \oplus S t^{s} \oplus \cdots \oplus S t^{(r-1) s}$.

(iv) $S$ is a maximal commutative associative subring of $\left(S / S_{0}, \sigma, d\right)$.

Proof. (i) is trivial as $S / S_{0}$ has constant rank $m$.

(ii) By our general theory on Petit algebras, we know that $\left(S / S_{0}, \sigma, d\right)$ is an algebra with center $S_{0}$ satisfying these properties, cf. [19, Theorem 4].

(iii) We know that $\operatorname{Nuc}_{r}\left(\left(S / S_{0}, \sigma, d\right)\right)=\{g \in R \mid \operatorname{deg}(g)<m$ and $f g \in R f\}$ by [19, Theorem 
4]. We have $\operatorname{Nuc}_{r}\left(\left(S / S_{0}, \sigma, d\right)\right) \cap S=S$. Since $d \in \operatorname{Fix}\left(\sigma^{s}\right)$ we can easily conclude that also $t^{s} \in \operatorname{Nuc}_{r}\left(\left(S / S_{0}, \sigma, d\right)\right)$. This implies that the finitely generated $S_{0}$-module $S \oplus S t^{s} \oplus$ $\cdots \oplus S t^{(r-1) s}$ lies in the right nucleus of $\left(S / S_{0}, \sigma, d\right)$. Since $S / S_{0}$ has constant rank $m$ by our assumptions, this submodule has constant rank $m r$. Now $\operatorname{Nuc}_{r}\left(\left(S / S_{0}, \sigma, d\right)\right)$ is an $S_{0}$-submodule of $\left(S / S_{0}, \sigma, d\right)$ and $\left(S / S_{0}, \sigma, d\right)$ has rank $m^{2}$. Hence comparing ranks we conclude that if $s$ is prime then either $r=m$ and $S_{f}$ is an associative algebra, or $r<m$, and $\mathrm{Nuc}_{r}\left(\left(S / S_{0}, \sigma, d\right)\right)=S \oplus S t^{s} \oplus \cdots \oplus S t^{(r-1) s}$.

If $S_{0}$ is a domain, we also obtain that $\operatorname{Nuc}_{r}\left(\left(S / S_{0}, \sigma, d\right)\right)=S \oplus S t^{s} \oplus \cdots \oplus S t^{(r-1) s}$. by the same proof as the one of [21, Proposition 3.2.3] (note that there this is proved for the opposite algebra).

(iv) This generalizes [25, Lemma 3.1] and follows immediately from Proposition 6 above: For all $d \in S^{\times}$, the centralizer of $S$ in $\left(S / S_{0}, \sigma, d\right)$ is $\operatorname{Cent}_{(D, \sigma, d)}(S)=S$.

Hence $S$ is always contained in the nucleus of $\left(S / S_{0}, \sigma, d\right)$ and if $S_{0}$ is a domain, then the nucleus of $\left(S / S_{0}, \sigma, d\right)$ is $S$.

Theorem 8. Let $(D, \sigma, d)$ be a nonassociative generalized cyclic Azumaya algebra of constant rank $n^{2} m^{2}, S_{0}=\operatorname{Fix}(\sigma) \cap C$.

(i) $(D, \sigma, d)$ is finitely generated as an $S_{0}$-module.

(ii) $(D, \sigma, d)$ is non-associative if and only if $d \in D \backslash S_{0}$. If $(D, \sigma, d)$ is not associative then $(D, \sigma, d)$ contains $D$ in its left and middle nucleus, and if $S_{0}$ is a domain, then $\operatorname{Nuc}_{l}((D, \sigma, d))=\operatorname{Nuc}_{m}((D, \sigma, d))=D$.

(iii) Let $s$ be the smallest integer such that $d \in \operatorname{Fix}\left(\sigma^{s}\right)$, then either $m=r s$ for some integer $r$ and the left $S_{0}$-module

$$
C \oplus C t^{s} \oplus \cdots \oplus C t^{(r-1) s}
$$

of constant rank rm lies in $\operatorname{Nuc}_{r}((D, \sigma, d))$ or $m=r s+b$ for two integers $r$ and $b$ with $0<b<s$ and the left $S_{0}$-module

$$
C \oplus C t^{s} \oplus \cdots \oplus C t^{r s}
$$

of constant rank $(r+1) m$ lies in $\operatorname{Nuc}_{r}((D, \sigma, d))$. In particular, $S_{0} \subseteq \operatorname{Nuc}((D, \sigma, d))$.

(iv) $D$ is separable over $S_{0}$.

Proof. (i) This is proved analogously to [22, Lemma 3.2].

(ii) By our general theory on Petit algebras, we know that $(D, \sigma, d)$ is an algebra with center $S_{0}$ and the nuclei as claimed [19, Theorem 4].

(iii) We have $\operatorname{Nuc}_{r}((D, \sigma, d))=\{g \in R \mid \operatorname{deg}(g)<m$ and $f g \in R f\}$. It is easy to check that $C \subseteq \operatorname{Nuc}_{r}((D, \sigma, d))$. Let $s$ be the smallest integer such that $d \in \operatorname{Fix}\left(\sigma^{s}\right)$. Then a straightforward calculation shows that $t^{s} \in \operatorname{Nuc}_{r}((D, \sigma, d))$. Hence $C \oplus C t^{s} \oplus \cdots \oplus C t^{(r-1) s} \subseteq$ $\operatorname{Nuc}_{r}((D, \sigma, d))$ if $m=r s$ and $C \oplus C t^{s} \oplus \cdots \oplus C t^{r s} \subseteq \operatorname{Nuc}_{r}((D, \sigma, d))$ if $m=r s+b$.

(iv) By construction, $(D, \sigma, d)$ is free of rank $m$ as a left $D$-module. Since $D$ is finitely generated as a $C$-module by assumption, and since $C$ is finitely generated as an $S_{0}$-module as it is a Galois extension, $(D, \sigma, d)$ is finitely generated as an $S_{0}$-module.

(iv) is trivial by the assumptions on $D$ and $C / \operatorname{Fix}(\sigma)$. 


\section{Automorphisms of nonassociative generalized Cyclic Azumaya algebras}

3.1. Nonassociative generalized cyclic Azumaya algebras. Let $D$ be an Azumaya algebra over $C$ of constant rank $n$ and $A=(D, \sigma, d)$ be a nonassociative generalized cyclic Azumaya algebra over $S_{0}=C \cap \operatorname{Fix}(\sigma)$. In the following $\sigma$ sometimes also stands for $\left.\sigma\right|_{C}$ to simplify notation.

For $k \in C$, we define $N_{C / S_{0}}: C \longrightarrow S_{0}$ via

$$
N_{C / S_{0}}(k)=\left(\prod_{l=0}^{m-1} \sigma^{l}(k)\right) .
$$

Some of the ring automorphisms of the skew polynomial ring $D[t ; \sigma]$ canonically induce algebra automorphisms of $(D, \sigma, d)$ :

Theorem 9. Let $A=(D, \sigma, d)$ be a nonassociative generalized cyclic Azumaya algebra. Let $\tau \in \operatorname{Aut}_{S_{0}}(D)$ be an algebra automorphism that commutes with $\sigma$. For all $k \in C^{\times}$such that

$$
\tau(d)=\left(\prod_{l=0}^{m-1} \sigma^{l}(k)\right) d
$$

define $H_{\tau, k}:(D, \sigma, d) \longrightarrow(D, \sigma, d)$ via

$$
\begin{gathered}
H_{\tau, k}\left(\sum_{i=0}^{m-1} x_{i} t^{i}\right)=\sum_{i=0}^{m-1} \tau\left(x_{i}\right)(k t)^{i} \\
=\tau\left(x_{0}\right)+\tau\left(x_{1}\right) k t+\tau\left(x_{2}\right) k \sigma(k) t^{2}+\cdots+\tau\left(x_{m-1}\right) k \sigma(k) \cdots \sigma^{m-2}(k) t^{m-1} .
\end{gathered}
$$

Then $H_{\tau, k}$ is an automorphism of $A$ that extends $\tau . H_{\tau, k}$ is canonically induced by a ring automorphism of $D[t ; \sigma]$.

(ii) For all $k \in C^{\times}$such that $N_{C / S_{0}}(k)=1$,

$$
H_{i d, k}\left(\sum_{i=0}^{m-1} a_{i} t^{i}\right)=a_{0}+\sum_{i=1}^{m-1} a_{i}\left(\prod_{l=0}^{i-1} \sigma^{l}(k)\right) t^{i}
$$

is an automorphism of $(D, \sigma, d)$ extending $i d_{D}$.

Proof. (i) Let $G$ be a ring automorphism of $D[t ; \sigma]$. Then for $h(t)=\sum_{i=0}^{m-1} b_{i} t^{i} \in D[t$; $\sigma]$ we have

$$
G(h(t))=\tau\left(b_{0}\right)+\sum_{i=i}^{m-1} \tau\left(b_{i}\right) \prod_{l=0}^{i-1} \sigma^{l}(k) t^{i}
$$

for some $\tau \in \operatorname{Aut}(D)$ such that $\sigma \circ \tau=\tau \circ \sigma$ and some $k \in C^{\times}$(the proof of [14, p. 75] works for $D[t ; \sigma])$. It is straightforward to see that for $\tau \in \operatorname{Aut}_{S_{0}}(D)$ and $S_{f}=(D, \sigma, d)$, we have $S_{f} \cong S_{G(f)}$ (cf. [16, Theorem 7] or [6, p. $55 \mathrm{ff}$.], the proofs also work when $D$ is not a division algebra). In particular, this means that if $k \in C^{\times}$satisfies (1) then $G(f(t))=\left(\prod_{l=0}^{m-1} \sigma^{l}(k)\right) f(t)=a f(t)$ with $a \in D^{\times}$being the product of the $\sigma^{l}(k)$, and so $G$ induces an isomorphism of $S_{f}$ with $S_{a f}=S_{f}$, i.e. an automorphism of $S_{f}=(D, \sigma, d)$.

(ii) follows from (i).

All the automorphisms of a nonassociative generalized cyclic Azumaya algebra $(D, \sigma, d)$ are canonically induced by ring automorphisms of the twisted polynomial ring $D[t ; \sigma]$, i.e. the maps $H_{\tau, k}$ are all possible automorphisms: 
Theorem 10. Let $A=(D, \sigma, d), d \in D^{\times}$, be a nonassociative generalized cyclic Azumaya algebra of constant rank $m^{2} n^{2}$ over $S_{0}$.

(i) Let $H \in \operatorname{Aut}_{S_{0}}(A)$. Then $H=H_{\tau, k}$ with

$$
H_{\tau, k}\left(\sum_{i=0}^{m-1} a_{i} t^{i}\right)=\tau\left(a_{0}\right)+\sum_{i=1}^{m-1} \tau\left(a_{i}\right)\left(\prod_{l=0}^{i-1} \sigma^{l}(k)\right) t^{i},
$$

for some $\tau \in \operatorname{Aut}_{S_{0}}(D)$ which commutes with $\sigma$, and some $k \in C^{\times}$such that $\tau(d)=$ $N_{C / S_{0}}(k) d$. Moreover, if $\tau \in \operatorname{Aut}_{S_{0}}(D)$ commutes with $\sigma$, and $k \in C^{\times}$such that $\tau(d)=$ $N_{C / S_{0}}(k) d$, then $H_{\tau, k}$ is an automorphism of $A$, and induced by an automorphism of $D[t ; \sigma]$.

(ii) For all $k \in C^{\times}$such that $N_{C / S_{0}}(k)=1$, id $\in \operatorname{Aut}(D)$ extends to an automorphism $H=H_{i d, k} \in \operatorname{Aut}_{S_{0}}(A)$,

$$
H_{i d, k}\left(\sum_{i=0}^{m-1} a_{i} t^{i}\right)=a_{0}+\sum_{i=1}^{m-1} a_{i}\left(\prod_{l=0}^{i-1} \sigma^{l}(k)\right) t^{i},
$$

The proof is similar to the one of [7, Theorem 6], but works also when the algebra is non-associative, as it does not rely on the right nucleus being $D$, whereas the proof of $[7$, Theorem 6] did.

Proof. (i) Let $H \in \operatorname{Aut}_{S_{0}}(A)$. Then $\left.H\right|_{D} \in \operatorname{Aut}_{S_{0}}(D)$, since $H$ leaves the commutator $\operatorname{Cent}_{(D, \sigma, d)}(C)$ invariant and we know that $\operatorname{Cent}_{(D, \sigma, d)}(C)=D$. (The argument in the proof of $[7$, Theorem 6$]$ uses instead that the right nucleus is invariant under $H)$.

Thus $\left.H\right|_{D}=\tau$ for some $\tau \in \operatorname{Aut}_{S_{0}}(D)$. Write $H(t)=\sum_{i=0}^{m-1} k_{i} t^{i}$ for some $k_{i} \in D$, then we have

$$
H(t z)=H(t) H(z)=\left(\sum_{i=0}^{m-1} k_{i} t^{i}\right) \tau(z)=\sum_{i=0}^{m-1} k_{i} \sigma^{i}(\tau(z)) t^{i},
$$

and

$$
H(t z)=H(\sigma(z) t)=\tau(\sigma(z)) \sum_{i=0}^{m-1} k_{i} t^{i}=\sum_{i=0}^{m-1} \tau(\sigma(z)) k_{i} t^{i}
$$

for all $z \in D$. Comparing the coefficients of $t^{i}$ yields

$$
k_{i} \sigma^{i}(\tau(z))=\tau(\sigma(z)) k_{i} \text { for all } i=\{0, \ldots, m-1\}
$$

for all $z \in D$. This implies that

$$
k_{i}\left(\sigma^{i}(\tau(z))-\tau(\sigma(z))\right)=0 \text { for all } i \in\{0, \ldots, m-1\}
$$

for all $z \in C$. Now $\sigma$ restricted to $C$ generates the Galois group of the cyclic Galois extension $C / S_{0}$ by assumption, and $\left.\tau\right|_{C}: C \longrightarrow C$ fixes $S_{0}$ by assumption, thus lies in this Galois group. Hence $\left.\tau\right|_{C}$ commutes with $\left.\sigma\right|_{C}$ and we obtain

$$
k_{i}\left(\sigma^{i}(\tau(z))-\sigma(\tau(z))\right)=0 \text { for all } i \in\{0, \ldots, m-1\}
$$

for all $z \in C$, therefore

$$
k_{i}\left(\sigma^{i-1}(w)-w\right)=0 \text { for all } i \in\{1, \ldots, m-1\}
$$

for all $w \in C$. As $\left.\sigma\right|_{C}$ has order $m$, we know that $\left.\sigma\right|_{C} \neq\left.\sigma^{i}\right|_{C}$ for all $1 \neq i \in\{0, \ldots, m-1\}$.

Since $C / S_{0}$ is Galois, we also know that the ideal of $C$ generated by $\left\{c-\sigma^{i}(c) \mid c \in C\right\}$ is all of $C$ by $\left[10\right.$, p. 80]. That means $k_{i}=0$ for all $1 \neq i \in\{0, \ldots, m-1\}$. For $i=1$, we 
obtain $k_{1} \tau(\sigma(z))=\tau(\sigma(z)) k_{1}$ for all $z \in D$, hence $k_{1} \in C$. This implies $H(t)=k t$ for some $k \in C^{\times}$.

Since

$$
H\left(z t^{i}\right)=H(z) H(t)^{i}=\tau(z)(k t)^{i}=\tau(z)\left(\prod_{l=0}^{i-1} \sigma^{l}(k)\right) t^{i},
$$

for all $i \in\{1, \ldots, m-1\}$ and all $z \in D, H$ has the form

$$
H_{\tau, k}: \sum_{i=0}^{m-1} a_{i} t^{i} \mapsto \tau\left(a_{0}\right)+\sum_{i=1}^{m-1} \tau\left(a_{i}\right)\left(\prod_{l=0}^{i-1} \sigma^{l}(k)\right) t^{i},
$$

for some $k \in C^{\times}$.

Comparing the constant terms in $H(t)^{m}=H\left(t^{m}\right)=H(d)$ implies

$$
\tau(d)=k \sigma(k) \cdots \sigma^{m-1}(k) d=N_{C / S_{0}}(k) d .
$$

The fact that $H_{\tau, k}$ is by assumption a multiplicative map forces $\sigma$ and $\tau$ to commute: $H_{\tau, k}(t \circ c)=H_{\tau, k}(t) H_{\tau, k}(c)$ for all $c \in D$ implies that

$$
H_{\tau, k}(t \circ c)=H_{\tau, k}(\sigma(c) t)=\tau(\sigma(c)) k t
$$

and

$$
H_{\tau, k}(t) H_{\tau, k}(c)=k t \circ \tau(c)=k \sigma(\tau(c)) t .
$$

Thus we obtain $\tau(\sigma(c)) k=k \sigma(\tau(c))$ for all $c \in D$ and some $k \in C^{\times}$, implying $\tau(\sigma(c))=$ $\sigma(\tau(c))$ for all $c \in D$.

By Theorem 9 all these maps are automorphisms induced by automorphisms of the skew polynomial ring, since $\sigma$ and $\tau$ commute.

(ii) For $\tau=i d_{D}, H$ has the form

$$
H_{i d, k}\left(\sum_{i=0}^{m-1} a_{i} t^{i}\right)=a_{0}+\sum_{i=1}^{m-1} a_{i}\left(\prod_{l=0}^{i-1} \sigma^{l}(k)\right) t^{i}
$$

for some $k \in C^{\times}$with $k \sigma(k) \cdots \sigma^{m-1}(k)=N_{C / S_{0}}(k)=1$ by (i).

It is clear that $H_{\tau, k}=H_{\rho, l}$ if and only if $\sigma=\rho$ and $k=l$, and that $H_{\tau, k} \circ H_{\rho, l}=H_{\tau \circ \rho, k l}$.

Corollary 11. (i) The subgroup of $S_{0}$-automorphisms of $(D, \sigma, d)$ extending $i d_{D}$ is isomorphic to

$$
\left\{k \in C^{\times} \mid N_{C / S_{0}}(k)=1\right\} .
$$

(ii) Suppose that $S_{0}$ contains an mth root of unity $\omega$. If $\tau$ has finite order $s$, then the cyclic subgroup $\left\langle H_{\tau, \omega}\right\rangle$ of $\operatorname{Aut}_{S_{0}}(A)$ generated by $H_{\tau, \omega}$ has order at most ms. (If $\tau$ has infinite order then this subgroup has infinite order.) In particular, $\left\langle H_{i d, \omega}\right\rangle$ is a cyclic subgroup of $\operatorname{Aut}_{S_{0}}((D, \sigma, d))$ of order at most $m$.

As a consequence of Theorem 10 and Corollary 11 we obtain all automorphisms of a nonassociative cyclic Azumaya algebra: 
Theorem 12. Let $A=\left(S / S_{0}, \sigma, d\right)$ be a nonassociative cyclic Azumaya algebra.

(i) Let $H \in \operatorname{Aut}_{S_{0}}(A)$. Then $H=H_{\tau, k}$ with

$$
H_{\tau, k}\left(\sum_{i=0}^{m-1} a_{i} t^{i}\right)=\tau\left(a_{0}\right)+\sum_{i=1}^{m-1} \tau\left(a_{i}\right)\left(\prod_{l=0}^{i-1} \sigma^{l}(k)\right) t^{i},
$$

for some $\tau \in \operatorname{Aut}_{S_{0}}(S)$ and some $k \in S^{\times}$such that $\tau(d)=N_{S / S_{0}}(k) d$. All maps $H_{\tau, k}$ where $\tau \in \operatorname{Aut}_{S_{0}}(S)$ and where $k \in S^{\times}$such that $\tau(d)=N_{S / S_{0}}(k) d$, are automorphisms of $A$.

(ii) For all $k \in S^{\times}$such that $N_{S / S_{0}}(k)=1$, id $d_{S}$ can be extended to an automorphism

$$
H_{i d, k}\left(\sum_{i=0}^{m-1} a_{i} t^{i}\right)=a_{0}+\sum_{i=1}^{m-1} a_{i}\left(\prod_{l=0}^{i-1} \sigma^{l}(k)\right) t^{i}
$$

in $\operatorname{Aut}_{S_{0}}(A)$.

(iii) The subgroup of $S_{0}$-automorphisms of $A$ extending id $d_{S}$ is isomorphic to

$$
\left\{k \in S^{\times} \mid N_{S / S_{0}}(k)=1\right\} .
$$

Proof. (i) Let $S / S_{0}$ be a cyclic Galois ring extension with $\operatorname{Gal}\left(S / S_{0}\right)=\langle\sigma\rangle$ of order $m, \sigma$ an automorphism of $S$. Then for all $\tau \in \operatorname{Gal}\left(S / S_{0}\right)$ and $k \in S^{\times}$such that

$$
\tau(d)=\left(\prod_{l=0}^{m-1} \sigma^{l}(k)\right) d
$$

the map $H_{\tau, k}:\left(S / S_{0}, \sigma, d\right) \longrightarrow\left(S / S_{0}, \sigma, d\right)$,

$$
H_{\tau, k}\left(\sum_{i=0}^{m-1} x_{i} t^{i}\right)=\sum_{i=0}^{m-1} \tau\left(x_{i}\right)(k t)^{i}
$$

is an automorphism of the nonassociative cyclic Azumaya algebra $\left(S / S_{0}, \sigma, d\right)$ that extends $\tau$ (choose $D=C$ in Theorem 9). Since all $\tau \in \operatorname{Aut}_{S_{0}}(S)$ commute with $\sigma$, we obtain all automorphisms this way by Theorem 10 .

(ii) and (iii) follow from (i) and Corollary 11.

Note that if $S_{0}$ contains an $m$ th root of unity $\omega$, and $\tau$ has finite order $s$, then the cyclic subgroup $\left\langle H_{\tau, \omega}\right\rangle$ of $\operatorname{Aut}_{S_{0}}\left(\left(S / S_{0}, \sigma, d\right)\right)$, again has order at most $m s$.

If $S_{0}$ has no non-trivial $m$ th root of unity, we obtain:

Theorem 13. Suppose $S_{0}$ has no non-trivial mth root of unity. Let $A=\left(S / S_{0}, \sigma, d\right)$ be a nonassociative cyclic algebra of degree $m$ where $d \in S^{\times}$is not contained in any proper subring of $S$. Then every $S$-automorphism of $A$ leaves $S_{0}$ fixed and

$$
\operatorname{Aut}_{S_{0}}(A) \cong \operatorname{ker}\left(N_{S / S_{0}}\right) .
$$

Proof. Every automorphism of $A$ has the form $H_{i d, k}$ : suppose that there exist $j \in\{1, \ldots, m-$ $1\}$ and $k \in S^{\times}$such that $H_{\sigma^{j}, k} \in \operatorname{Aut}_{S_{0}}(A)$. This implies $H_{\sigma^{j}, k}^{2}=H_{\sigma^{j}, k} \circ H_{\sigma^{j}, k} \in \operatorname{Aut}_{S_{0}}(A)$ and

$$
H_{\sigma^{j}, k}^{2}\left(\sum_{i=0}^{m-1} x_{i} t^{i}\right)=\sigma^{2 j}\left(x_{0}\right)+\sum_{i=1}^{m-1} \sigma^{2 j}\left(x_{i}\right)\left(\prod_{q=0}^{i-1} \sigma^{j+q}(k) \sigma^{q}(k)\right) t^{i}
$$


Now $H_{\sigma^{j}, k}^{2}$ must have the form $H_{\sigma^{2 j}, l}$ for some $l \in S^{\times}$. Comparing (2) and (4) yields $l=k \sigma^{j}(k)$. Similarly, $H_{\sigma^{j}, k}^{3}=H_{\sigma^{3 j}, s} \in \operatorname{Aut}_{S_{0}}(A)$ where $s=k \sigma^{j}(k) \sigma^{2 j}(k)$. Continuing in this manner the automorphisms $H_{\sigma^{j}, k}, H_{\sigma^{2 j}, l}, H_{\sigma^{3 j}, s}, \ldots$ all satisfy (1) implying that

$$
\begin{aligned}
\sigma^{j}(d) & =N_{S / S_{0}}(k) d, \\
\sigma^{2 j}(d) & =N_{S / S_{0}}\left(k \sigma^{j}(k)\right) d=N_{S / S_{0}}(k)^{2} d, \\
\vdots & \vdots \\
d=\sigma^{n j}(d) & =N_{S / S_{0}}(k)^{n} d,
\end{aligned}
$$

where $n=m / \operatorname{gcd}(j, m)$ is the order of $\sigma^{j}$. Note that $\sigma^{i j}(d) \neq d$ for all $i \in\{1, \ldots, n-1\}$ since $d$ is not contained in any proper subring of $S$. Therefore $N_{S / S_{0}}(k)^{n}=1$ and $N_{S / S_{0}}(k)^{i} \neq 1$ for all $i \in\{1, \ldots, n-1\}$ by $(5)$, i.e. $N_{S / S_{0}}(k)$ is a primitive $n$th root of unity, thus also an $m$ th root of unity, a contradiction. This proves the assertion.

3.2. Associative generalized cyclic Azumaya algebras. In the associative setting, the previous results show that all automorphisms of a generalized cyclic Azumaya algebra $(D, \sigma, d)$ are induced by automorphisms of $D[t ; \sigma]$ :

Corollary 14. Let $A=(D, \sigma, d)$ be a generalized cyclic Azumaya algebra, i.e. $d \in S_{0}^{\times}$.

(i) Every $\tau \in \operatorname{Aut}_{S_{0}}(D)$ that commutes with $\sigma$ can be extended to an automorphism

$$
H_{\tau, k}\left(\sum_{i=0}^{m-1} a_{i} t^{i}\right)=\sum_{i=0}^{m-1} \tau\left(a_{i}\right)(k t)^{i}=\tau\left(a_{0}\right)+\sum_{i=1}^{m-1} \tau\left(a_{i}\right)\left(\prod_{l=0}^{i-1} \sigma^{l}(k)\right) t^{i}
$$

in $\operatorname{Aut}_{S_{0}}(A)$ for some $k \in C^{\times}$such that $N_{C / S_{0}}(k)=1$. All maps $H_{\tau, k}$ where $\tau \in \operatorname{Aut}_{S_{0}}(D)$ commutes with $\sigma$ and where $k \in C^{\times}$such that $N_{C / S_{0}}(k)=1$ are automorphisms of $A$.

(ii) The subgroup of $S_{0}$-automorphisms extending some fixed $\tau \in \operatorname{Aut}_{S_{0}}(D)$ is isomorphic to

$$
\left\{k \in C^{\times} \mid N_{C / S_{0}}(k)=1\right\} .
$$

(iii) Suppose that $S_{0}$ contains a primitive mth root of unity $\omega$. If $\tau$ has finite order $s$, then the cyclic subgroup $\left\langle H_{\tau, \omega}\right\rangle$ of $\operatorname{Aut}_{S_{0}}(A)$ generated by $H_{\tau, \omega}$ has order ms. (If $\tau$ has infinite order then this subgroup has infinite order.) Furthermore, $\left\langle H_{i d, \omega}\right\rangle$ is a cyclic subgroup of $\operatorname{Aut}_{S_{0}}((D, \sigma, d))$ of order $m$.

(iv) $\left\{\tau \in \operatorname{Aut}_{S_{0}}(D) \mid \tau \circ \sigma=\sigma \circ \tau\right\}$ is isomorphic to a subgroup of $\operatorname{Aut}_{S_{0}}(A)$.

Proof. It remains to show (iv), which holds since we have the canonical extension $H_{\tau, 1}$ for each $\tau \in \operatorname{Aut}_{S_{0}}(A)$ that commutes with $\sigma$.

Corollary 15. Let $A=\left(S / S_{0}, \sigma, d\right), d \in S_{0}^{\times}$, be a cyclic Azumaya algebra. Then every $\tau \in \operatorname{Aut}_{S_{0}}(S)$ can be extended to an automorphism

$$
H_{\tau, k}\left(\sum_{i=0}^{m-1} a_{i} t^{i}\right)=\tau\left(a_{0}\right)+\sum_{i=1}^{m-1} \tau\left(a_{i}\right)\left(\prod_{l=0}^{i-1} \sigma^{l}(k)\right) t^{i}
$$

in Aut $_{S_{0}}(A)$ for some $k \in S^{\times}$such that $N_{S / S_{0}}(k)=1$. All maps $H_{\tau, k}$ where $\tau \in \operatorname{Aut}_{S_{0}}(S)$ and where $k \in S^{\times}$such that $N_{S / S_{0}}(k)=1$ are algebra automorphisms of $A$.

In particular, $\operatorname{Aut}_{S_{0}}(S)=\langle\sigma\rangle \subseteq \operatorname{Aut}_{S_{0}}(A)$. 
These are all possible automorphisms. So there is a bijection between the set of automorphisms of $A=\left(S / S_{0}, \sigma, d\right)$ and the set

$$
\left\{(\tau, k) \mid \tau \in \operatorname{Aut}_{S_{0}}(A), k \in S^{\times} \text {with } N_{S / S_{0}}(k)=1\right\}
$$

and for each $\tau \in \operatorname{Aut}_{S_{0}}(A)$, there are either infinitely many (if the set of norm one elements in $S$ is infinite) or $\left|\left\{k \in S^{\times} \mid N_{S / S_{0}}(k)=1\right\}\right|$ different possible extensions.

\section{INNER AUTOMORPHISMS}

We now consider the inner automorphisms of nonassociative generalized cyclic Azumaya algebras.

Theorem 16. (a) Let $A=(D, \sigma, d)$ be a nonassociative generalized cyclic Azumaya algebra. Let $k \in C$ such that there is $c \in C^{\times}$with $k=c^{-1} \sigma(c)$.

(i) $H_{\tau, k}=G_{c} \circ H_{\tau, 1}$ (note that $H_{\tau, 1}$ is not necessarily an automorphism here, just a map).

(ii) The automorphism $H_{i d, k}$ of $A$ is the inner automorphism

$$
G_{c}\left(\sum_{i=0}^{m-1} a_{i} t^{i}\right)=\left(c^{-1} \sum_{i=0}^{m-1} a_{i} t^{i}\right) c
$$

(b) Let $A=\left(S / S_{0}, \sigma, d\right)$ be a nonassociative cyclic Azumaya algebra. Let $k \in S$ such that there is $c \in S^{\times}$with $k=c^{-1} \sigma(c)$.

(i) $H_{\tau, k}=G_{c} \circ H_{\tau, 1}$ (note that $H_{\tau, 1}$ again is not necessarily an automorphism here, just a map).

(ii) The automorphism $H_{i d, k}$ of $A$ is the inner automorphism

$$
G_{c}\left(\sum_{i=0}^{m-1} a_{i} t^{i}\right)=\left(c^{-1} \sum_{i=0}^{m-1} a_{i} t^{i}\right) c
$$

Proof. (a) (i) For $k \in C$ such that $k=c^{-1} \sigma(c)$ for some $c \in C^{\times}$, we have

$$
k \sigma(k) \cdots \sigma^{i-1}(k)=c \sigma^{i}(c), \quad i=1 \ldots, m-1,
$$

hence

$$
G_{c}\left(\sum_{i=0}^{m-1} \tau\left(a_{i}\right) t^{i}\right)=\left(c^{-1} \sum_{i=0}^{m-1} \tau\left(a_{i}\right) t^{i}\right) c=\sum_{i=0}^{m-1} \tau\left(a_{i}\right) c^{-1} \sigma^{i}(c) t^{i}=H_{\tau, k}\left(\sum_{i=0}^{m-1} a_{i} t^{i}\right)
$$

with the last equality holding because of $\Pi_{l=0}^{i-1} \sigma^{l}\left(c^{-1} \sigma(c)\right)=c^{-1} \sigma^{i}(c)$.

(ii) follows from (i) and (b) from (a).

A similar result to Theorem 16 (a) (i) was proved for automorphisms of (associative) $G$-Azumaya algebras over a connected commutative ring $R$ with a primitive $s$ th root of unity in [5] when $G$ is a finite abelian group of finite order $n$ and exponent $s$, provided that $\operatorname{Pic}_{s}(R)$ is trivial and $n$ a unit in $R$.

Let us look at the associative setting: 
Theorem 17. (a) Let $A=(D, \sigma, d)$ be a generalized cyclic Azumaya algebra.

(i) Let $k \in C^{\times}$such that there is $c \in C^{\times}$with $k=c^{-1} \sigma(c)$. Then $H_{\tau, k}=G_{c} \circ H_{\tau, 1}$.

(ii) $H_{\sigma, 1}=G_{t^{-1}}$ is an inner automorphism. Moreover,

$$
\left\{G_{c t^{-j}} \mid c \in C^{\times}, 0 \leq i \leq m-1\right\}
$$

is a subgroup of $\operatorname{Aut}_{S_{0}}(A)$ of inner automorphisms.

(b) Let $A=\left(S / S_{0}, \sigma, d\right)$ be a cyclic Azumaya algebra.

(i) Let $k \in S^{\times}$such that there is $c \in S^{\times}$with $k=c^{-1} \sigma(c)$. Then $H_{\tau, k}=G_{c} \circ H_{\tau, 1}$.

(ii) $\left\{G_{c t^{-j}} \mid c \in S^{\times}, 0 \leq i \leq m-1\right\}$ is a subgroup of $\operatorname{Aut}_{S_{0}}(A)$ of inner automorphisms.

Proof. (a) (i) follows from Theorem 16.

(ii) We know that $t^{m}=d$ in $A$. This means that $d^{-1} t^{m-1}$ is the inverse of $t$, since $d \in S_{0}$. We have

$$
G_{t^{-1}}\left(\sum_{i=0}^{m-1} a_{i} t^{i}\right)=\sum_{i=0}^{m-1} t a_{i} t^{i}\left(t^{m-1} d^{-1}\right)=d^{-1} \sum_{i=0}^{m-1} \sigma\left(a_{i}\right) t^{i} d=H_{\sigma, 1}\left(\sum_{i=0}^{m-1} a_{i} t^{i}\right) .
$$

Thus also $H_{\sigma^{j}, 1}=G_{t^{-j}}$ is an inner automorphism for all integers $j, 0 \leq j \leq m-1$, and so is $G_{c} \circ H_{\sigma^{j}, 1}=H_{\sigma^{j}, c^{-1} \sigma(c)}=G_{c t^{-j}}$ for all $c \in C^{\times}$. The rest of the assertion is trivial.

All of (b) follows from (a).

Corollary 18. (i) Let $(D, \sigma, d)$ be a generalized cyclic Azumaya algebra. If there exists an analogue of Hilbert's Theorem 90 for the ring extension $C / S_{0}$ (i.e., for every $k \in C$ with $N_{C / S_{0}}(k)=1$ there is $c \in C^{\times}$such that $\left.k=c^{-1} \sigma(c)\right)$, then

$$
\begin{aligned}
\operatorname{Aut}_{S_{0}}((D, \sigma, d))= & \left\{H_{\tau, k} \mid \tau \in \operatorname{Aut}_{S_{0}}(D), \sigma \circ \tau=\tau \circ \sigma, k \in C^{\times} \text {such that } N_{C / S_{0}}(k)=1\right\} \\
& =\left\{G_{c} \circ H_{\tau, 1} \mid \tau \in \operatorname{Aut}_{S_{0}}(D), \sigma \circ \tau=\tau \circ \sigma, c \in C^{\times}\right\} .
\end{aligned}
$$

(ii) If there exists an analogue of Hilbert's Theorem 90 for the cyclic Galois ring extension $S / S_{0}$, then the cyclic Azumaya algebra $\left(S / S_{0}, \sigma, d\right)$ has the automorphism group

$$
\operatorname{Aut}_{S_{0}}\left(\left(S / S_{0}, \sigma, d\right)\right)=\left\{G_{c t^{-j}} \mid c \in S^{\times}, 0 \leq i \leq m-1\right\} .
$$

Proof. (i) The first equality is clear by Theorem 10. By assumption, we can write $H_{\tau, k}=$ $G_{c}\left(H_{\tau, 1}\right)$ for $k=\sigma(c) c^{-1}$, that is

$$
H_{\tau, k}\left(\sum_{i=0}^{m-1} a_{i} t^{i}\right)=\left(c^{-1} \sum_{i=0}^{m-1} \tau\left(a_{i}\right) t^{i}\right) c
$$

which implies the second equality.

(ii) We know that $\operatorname{Aut}_{S_{0}}\left(\left(S / S_{0}, \sigma, d\right)\right)=\left\{H_{\sigma^{j}, k} \mid 0 \leq j \leq m-1, k \in S^{\times}\right.$such that $N_{S / S_{0}}(k)=$ 1 . Now we also have $H_{\sigma^{j}, l}=G_{c} \circ H_{\sigma^{j}, 1}$ for $l=\sigma(c) c^{-1}$, and $H_{\sigma^{j}, 1}=G_{t^{-1}} \circ G_{t^{-1}} \circ \cdots \circ G_{t^{-1}}=$ $G_{t^{-j}}$, thus $H_{\sigma^{j}, l}=G_{c} \circ G_{t^{-j}}=G_{c t^{-j}}$ is an inner automorphism. This implies the assertion. 
4.1. The automorphisms of central simple algebras. In this section let $D$ be a division algebra which is finite-dimensional over its center $F=\mathrm{C}(D)$ and $\sigma \in \operatorname{Aut}(D)$ such that $\left.\sigma\right|_{F}$ has finite order $m$ and fixed field $F_{0}=\operatorname{Fix}(\sigma) \cap F$. Thus $F / F_{0}$ is automatically a cyclic Galois field extension of degree $m$ with $\operatorname{Gal}\left(F / F_{0}\right)=\left\langle\left.\sigma\right|_{F}\right\rangle$. For all $d \in F_{0}^{\times}, A=(D, \sigma, d)$ is a generalized cyclic central simple algebra over $F_{0}$, cf. [13].

As an immediate consequence of our results, the automorphisms of a generalized cyclic algebra over a field are induced by ring automorphisms of the ring $D[t ; \sigma]$ used in their construction. More precisely, they can be described as the composition of an inner automorphism $G_{c}, c \in F^{\times}$, with the canonical extension $H_{\tau, 1}$ of some $\tau \in \operatorname{Aut}_{F_{0}}(D)$ which commutes with $\sigma$ :

Corollary 19. (i) Let $A=(D, \sigma, d)$ be a central simple algebra over $F_{0}$, then

$$
\begin{gathered}
\operatorname{Aut}_{F_{0}}(A)=\left\{H_{\tau, k} \mid \tau \in \operatorname{Aut}_{F_{0}}(D), \sigma \circ \tau=\tau \circ \sigma, k \in F^{\times} \text {such that } N_{F / F_{0}}(k)=1\right\} \\
=\left\{G_{c} \circ H_{\tau, 1} \mid \tau \in \operatorname{Aut}_{F_{0}}(D), \sigma \circ \tau=\tau \circ \sigma, c \in F^{\times}\right\} .
\end{gathered}
$$

(ii) Let $A=(K / F, \sigma, d)$ be a cyclic algebra over $F$ of degree $m$. Then

$$
\operatorname{Aut}_{F}(A)=\left\{G_{c t^{-j}} \mid c \in K^{\times}, 0 \leq j \leq m-1\right\} .
$$

\section{REFERENCES}

[1] A. A. Albert, Cyclic fields of degree $p^{n}$ over $F$ of characteristic p. Bull. AMS 40 (1934), 625-631.

[2] A. A. Albert, Modern higher algebra. Chicago 1937, Chapter IX, 192-208.

[3] A. S. Amitsur, Non-commutative cyclic fields. Duke Math. J. 21 (1954), 87105.

[4] E. Artin, O. Schreier, Über eine Kennzeichnung der reell algebraischen Körper. Abh. Math. Seminar der Hamburgischen Universitäten, 5 (1927), 225-231.

[5] M. Beattie, Automorphisms of G-Azumaya algebras. Canad. J. Math. 37 (6) (1985) 1047-1058.

[6] C. Brown, Petit algebras and their automorphisms. PhD Thesis, University of Nottingham 2018. Online at arXiv:1806.00822 [math.RA]

[7] C. Brown, S. Pumplün, Nonassociative cyclic extensions of fields and central simple algebras. J. Pure Applied Algebra 223 (6) 2019, 2401-2412.

[8] C. Brown, S. Pumplün, How a nonassociative algebra reflects the properties of a skew polynomial. Online at arXiv:1806.04537v1 [math.RA]

[9] S.U. Chase, D.K. Harrison, A. Rosenberg, Galois theory and Galois cohomology of commutative rings. Mem. Amer. Math. Soc. 52 (1965), 15-33.

[10] F. DeMeyer, E. Ingraham, "Separable algebras over commutative rings". Lecture Notes in Mathematics 181 (1971) Springer-Verlag, Berlin-New York.

[11] M. Ferrero, A. Paques, Galois theory of commutative rings revisited. Beiträge Algebra Geom. 38 (2) (1997), 399-410.

[12] S. Ikehata, G. Szeto, On H-skew polynomial rings and Galois extensions. Rings, extensions, and cohomology (Evanston, IL, 1993), 113-121. Lecture Notes in Pure and Appl. Math., 159, Dekker, New York, 1994.

[13] N. Jacobson, "Finite-dimensional division algebras over fields." Springer Verlag, Berlin-Heidelberg-New York, 1996.

[14] K. Kishimoto, On cyclic extensions of simple rings. J. Fac. Sci. Hokkaido Univ. Ser. I 19 (1966), 74-85.

[15] M.-A. Knus, "Quadratic and hermitian forms over rings". Springer Verlag, Berlin-Heidelberg-New York, 1991.

[16] M. Lavrauw, J. Sheekey, Semifields from skew-polynomial rings. Adv. Geom. 13 (4) (2013), 583-604.

[17] J.-C. Petit, Sur certains quasi-corps généralisant un type d'anneau-quotient. Séminaire Dubriel. Algèbre et théorie des nombres 20 (1966 - 67), 1-18. 
[18] J.-C. Petit, Sur les quasi-corps distributifes à base momogène. C. R. Acad. Sc. Paris 266 (1968), Série A, 402-404.

[19] S. Pumplün, Finite nonassociative algebras obtained from skew polynomials and possible applications to $(f, \sigma, \delta)$-codes. Adv. Math. Comm. 11 (3) (2017), 615-634. doi:10.3934/amc.2017046

[20] R. D. Schafer, "An Introduction to Nonassociative Algebras." Dover Publ., Inc., New York, 1995.

[21] A. Steele, Some new classes of division algebras and potential applications to space-time block coding. PhD Thesis, University of Nottingham 2013, online at eprints.nottingham.ac.uk/13934

[22] G. Szeto, L. Xue, On splitting rings for Azumaya skew polynomial rings. Algebra Colloq. 8 (1) (2001), 25-32.

[23] G. Szeto, Splitting rings for Azumaya quaternion algebras. Brauer groups in ring theory and algebraic geometry (Wilrijk, 1981), Lecture Notes in Math., 917, 118-125, Springer, Berlin-New York, 1982.

[24] G. Szeto, On Azumaya crossed products. Bull. Malays. Math. Sci. Soc. (2) 35 (2012), no. 2A, 529-535.

[25] G. Szeto, A characterization of a cyclic Galois extension of commutative rings. J. Pure Appl. Algebra 16 (1980), no. 3, 315-322.

[26] S. Parimala, R. Sridharan, Projective modules over quaternion algebras. J. Pure Applied Algebra 9 (1977), 181 - 193.

[27] G. P. Wene, Inner automorphisms of semifields. Note Mat. 29 (1) (2009), 231-242.

[28] E. Witt, Zyklische Körper und Algebren der Charakteristik p vom Grad p ${ }^{n}$. J. Reine Angew. Math. 176 (1936), 126-140.

Email address: susanne.pumpluen@nottingham.ac.uk

School of Mathematical Sciences, University of Nottingham, University Park, Nottingham NG7 2RD, UNITED Kingdom 